\title{
EPIDEMIOLOGIA, DIAGNÓSTICO E TRATAMENTO NUTRICIONAL EM PORTADORES DE DIABETES MELLITUS
}

\author{
Girlane Nayara do Nascimento Santana'; Marcella de Arruda Moreira² \\ 1.Discente do Curso de Graduação em Nutrição do Centro Universitário Maurício de Nassau; \\ 2. Docente do Curso de Graduação em Nutrição em Nutrição do Centro Universitário Maurício de Nassau; Mestre em Nutrição \\ em Saúde Pública - UFPE;
}

\section{RESUMO}

O aumento na prevalência de diabetes mellitus é um dos motivos mais preocupantes em relação à saúde pública. Diante disso a terapia nutricional torna-se uma importante aliada no controle desta doença crônica. Este trabalho, portanto, trata-se de uma revisão de literatura que evidencia a importância de uma intervenção nutricional adequada. Tendo por base fontes primárias indexadas nas bases de dados Scielo, LILACS, Medline, entre outros, referente a um período de 2003 a 2012. A diabetes mellitus é uma doença crônica de etiologia múltipla, ocasionada pela ausência de insulina ou pela incapacidade da insulina em exercer adequadamente sua função. $O$ presente trabalho teve como objetivo descrever a epidemiologia, diagnóstico e a importância da terapia nutricional no DM tipo 1, tipo 2 e gestacional que acometem a população. A nutrição deve ser estabelecida de acordo com as quantidades adequadas de macronutrientes e micronutrientes prescritos, de forma individualizada, respeitando as fases da vida, hábitos alimentares, diagnóstico e aspectos socioculturais. Diante desses achados, o sucesso para o controle da diabetes mellitus esta no conhecimento sobre as recomendações para uma dieta adequada que possibilitem ao individuo diabético melhora na glicemia e diminuição nas complicações ocasionadas pela doença. A terapia nutricional torna-se portanto, importante por promover melhora na qualidade de vida.

Palavras-chave: Diabetes mellitus. Diagnóstico. Dietoterapia.

\begin{abstract}
The increased prevalence of diabetes mellitus is one of the reasons most worrying in relation to public health. Given this nutritional therapy becomes an important ally in controlling this chronic disease. This work, therefore, it is a literature review that highlights the importance of adequate nutritional intervention. Based on primary sources indexed in databases SciELO, LILACS, Medline, among others, relating to a period from 2003 to 2012. Diabetes mellitus is a chronic disease of multiple etiology, caused by lack of insulin or the inability of insulin to perform its function properly. This study aimed to describe the epidemiology, diagnosis and the importance of nutrition therapy in diabetes mellitus type 1, type 2 and gestational affecting the population. Nutrition should be established in accordance with adequate amounts of macronutrients and micronutrients prescribed individually, respecting the stages of life, eating habits, diagnosis and sociocultural aspects. Given these findings, the success for the control of diabetes mellitus in this knowledge about the recommendations for a proper diet that allow the individual diabetic improvement in glucose and decrease in complications caused by the disease. Nutritional therapy becomes therefore important for improving the quality of life.
\end{abstract}

Keywords: Diabetes mellitus. Diagnosis. Diet Therapy

\section{INTRODUÇÃO}


Diabetes mellitus (DM) é uma doença metabólica caracterizada por níveis elevados de glicose (hiperglicemia), resultante da deficiência e/ou ação da secreção de insulina. ${ }^{1}$ É uma doença altamente limitante, que pode resultar em várias complicações, dentre elas, podemos referir: a cegueira, amputações, nefropatias, complicações cardiovasculares e encefálicas, acarretando assim, prejuízos à capacidade funcional, autonomia e qualidade de vida do indivíduo. ${ }^{2}$

O DM é classificado de acordo com sua etiologia. ${ }^{3}$ Os três tipos clássicos de diabetes são: diabetes tipo 1, diabetes tipo 2 e diabetes gestacional. No diabetes tipo 1 (DMT1) o organismo não produz insulina, devido a destruição das células $\beta$ das ilhotas pancreáticas, resultante de um processo imunológico. $O$ tratamento com insulina nesse caso é fundamental para a manutenção da vida. ${ }^{4}$ Já no diabetes tipo 2 (DMT2) ou o corpo não produz insulina suficiente ou as células "ignoram" a insulina. ${ }^{5} \mathrm{O}$ tratamento dos pacientes com DMT2 consiste em "modificações do estilo de vida", associada ou não a agentes hipoglicemiantes orais. ${ }^{4} \mathrm{O}$ diabetes gestacional refere-se a qualquer intolerância à glicose com início ou diagnóstico no período gestacional, na maioria dos casos a glicemia retorna ao normal depois do parto. ${ }^{3}$

De acordo com um estudo realizado pela Sociedade Brasileira de Diabetes (SBD), estima-se que o número de diabéticos no Brasil chegue a 12.054.827 (doze milhões cinquenta e quatro mil oitocentos e vinte quatro). ${ }^{6} \mathrm{O}$ DM já afeta cerca de 246 milhões de pessoas em todo o mundo, com projeção para que esse número até 2025 chegue a 380 milhões.

Este estudo torna-se relevante devido à magnitude do DM, que cresce rapidamente em países pobres e em desenvolvimento, refletindo de forma negativa por estar relacionada à morbimortalidade precoce, além de afligir a previdência social por atingir pessoas em plena atividade produtiva. As consequências do DM afetam os sistemas de saúde em todo o mundo, tornando-se um desafio. Sendo assim, essa revisão tem como objetivo descrever a epidemiologia, diagnóstico e a importância da terapia nutricional no DM tipo 1, tipo 2 e gestacional que acometem a população.

\section{METODOLOGIA}


A fim de realizar uma revisão da literatura, procedeu-se à pesquisa de artigos científicos nos bancos de dados MedLine, Lilacs (Literatura Latino-Americana e do Caribe em Ciências da Saúde) e Scielo (Scientific Electronic Library Online), segundo os descritores em ciências da saúde (DeCS): diabetes mellitus, diagnóstico e dietoterapia.

A partir de uma leitura prévia dos resumos dos artigos encontrados, foram selecionados os mais relevantes, publicados no período entre 2003 a 2012 considerando estes os critérios de inclusão da presente pesquisa. Assim, após o estudo da íntegra dos artigos, compreendendo as principais ideias expostas, e a interpretação dos dados, a amostra resultou em 25 artigos publicados em periódicos indexados, na língua portuguesa, 2 bibliografias de livros e 1 cartilha e 2 diretrizes. Segundo informações colhidas para o presente estudo, permitiu-se a formulação de questões norteadoras sobre a terapia nutricional do DM.

\section{REFERENCIAL TEÓRICO}

\section{1- Etiologia e epidemiologia}

Em 1921 um dos mais importantes diabetologistas da época, Elliot P. Joslin definiu diabetes como uma doença crônica não contagiosa, que evoluía sem dor e passível de ser tratada cronicamente. 7 Diabete provém do termo "diabeinein", que significa "fluir através de um sifão", que descreve a presença de diurese e sede excessivas em alguns indivíduos, comparando-os com um sifão, cujos líquidos entram e saem rapidamente. ${ }^{3}$

Quando a educação em DM passou a fazer parte do tratamento nos anos de 1970, a morbimortalidade da população alvo melhorou sensivelmente. Em 1980, o estímulo ao autocontrole e à educação em DM refletiu na melhora do controle e na maior flexibilidade no tratamento da doença. Na década de 1990, surgiram os análogos de insulina, que junto a outros fatores passaram a oferecer um controle mais rigoroso da glicemia, melhorando a qualidade de vida dos portadores de DM1. Da mesma maneira, o mesmo ocorreu com o uso de antidiabéticos orais mais específicos, atendendo as necessidades individuais de pacientes com (DM2). ${ }^{7}$ 
A classificação proposta pela Organização Mundial da Saúde (OMS) e pela Associação Americana de Diabetes (ADA) inclui quatro classes clinicas: DM tipo 1, DM tipo 2, outros tipos específicos de DM e diabetes mellitus gestacional (DMG). ${ }^{8}$

O DM1 geralmente é diagnosticado em crianças e adultos jovens. Neste tipo de diabetes o organismo não produz insulina, hormônio necessário para converter sacarose, amidos e outros alimentos em energia necessária para a vida. Já no DM2, que é a forma mais comum de diabetes, ou o corpo não produz insulina suficiente ou as células ignoram a insulina. $O$ diabetes gestacional geralmente surge em torno da 24a semana, porém seu diagnóstico não significa a presença de diabetes antes de concebido ou a continuidade após o parto. ${ }^{5}$

O número de diabéticos está aumentando em consequência do crescimento e do envelhecimento populacional, à maior urbanização, à crescente prevalência de obesidade e sedentarismo, bem como à maior sobrevida do paciente com DM. Quantificar a prevalência de DM e o número de indivíduos diabéticos, no presente e no futuro, é importante para permitir uma forma racional de planejamento e alocação de recursos. ${ }^{8}$

As evidências apontam a relação entre a qualidade da alimentação e os riscos de desenvolver o DM. Mudanças observadas no consumo alimentar no Brasil, como o aumento da densidade energética, o maior consumo de carnes, leite e derivados ricos em gorduras, além da redução do consumo de cereais, frutas, verduras e legumes são importantes fatores de risco para o surgimento do DM, independentemente do índice de massa corporal (IMC). ${ }^{9}$ A nutrição, portanto, desempenha um papel essencial tanto na prevenção ${ }^{8}$ como controle do $\mathrm{DM}$, pois este se encontra diretamente relacionado ao metabolismo de carboidratos $(\mathrm{CHO})$, proteínas (PTN) e lipídios (LIP). ${ }^{10}$

O número de internações por DM no Brasil no ano de 2010 chegou a 148.452, em 2008 foram registrados 131.734. O valor destas internações até 2011 foi estimado em 87,9 milhões de reais. Quando se analisa a mortalidade (óbitos/ 100.000 habitantes) por DM nos últimos anos constata-se um crescente aumento, visto que em 2006 o percentual foi de $24,1 \%$, e em 2010 chegou a $28,8 \%{ }^{11}$ Entretanto, já foi estabelecido que o número de óbitos seja consideravelmente subestimado. ${ }^{8}$ 
Frequentemente o DM não é mencionado na declaração de óbito pelo fato de serem suas complicações, particularmente as cardiovasculares e cerebrovasculares, as causas da morte, sendo estas cadastradas nas estatísticas sobre mortalidade. Segundo informações que configurem essa doença como uma epidemia, sugerem cerca de 4 milhões de óbitos anuais relacionados a presença de DM, com importante contribuição de complicações cardiovasculares, correspondendo aproximadamente $9 \%$ do total mundial de mortes. ${ }^{8}$

No Brasil, de acordo com o Vigitel 2011 (Sistema de Monitoramento de Fatores de Risco e Proteção para Doenças Crônicas Não Transmissíveis), a ocorrência na população adulta (acima de 18) com diagnóstico médico referido para DM, em 26 capitais do Brasil e Distrito Federal é de 5,6\%, sendo que esta prevalência aumenta com a idade, atingindo $21,6 \%$ da população com idade superior a 65 anos. Estima-se que a proporção de diabetes não diagnosticada em diversos países no mundo está entre $30 \%$ e $60 \%{ }^{11}$

\section{2- Diagnóstico e quadro clínico}

Durante décadas, o diagnóstico de DM foi realizado com base em critérios da glicose plasmática em jejum e do Teste Oral de Tolerância à Glicose (TOTG). Em 2010, a ADA aprovou o teste da hemoglobina glicada (A1C) para o diagnóstico dessa patologia. Assim sendo, os critérios diagnósticos do DM baseiam-se nos valores descritos na tabela abaixo:12

Tabela 1. Critérios de diagnóstico do diabetes mellitus.

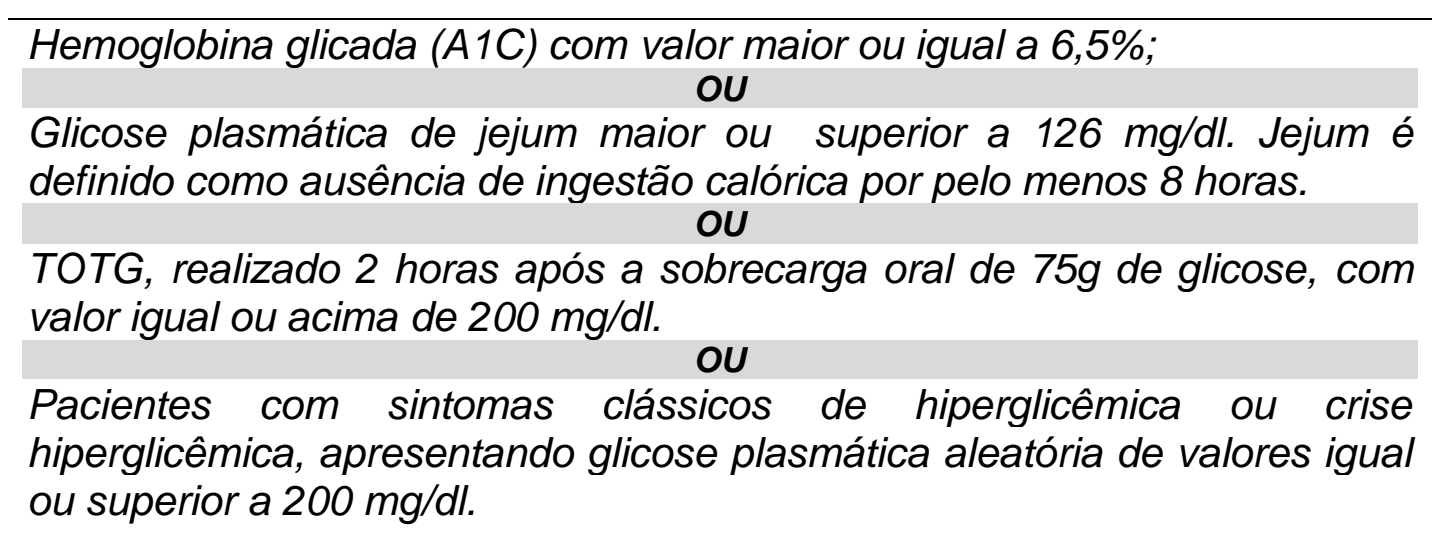

Fonte: Standards of Medical Care in Diabetes-2012

O procedimento diagnóstico recomendado pela OMS e pela ADA para 0 diagnóstico de DMG é o teste de sobrecarga com $75 \mathrm{~g}$ de glicose, realizado entre 24 e 28 semanas de gestação. Porém, se o rastreamento for positivo no primeiro 
trimestre, ou a gestante apresentar vários fatores de risco, este teste pode ser realizado mais precocemente. Os pontos de corte recomendados para o diagnóstico do diabetes gestacional são de $92 \mathrm{mg} / \mathrm{dL}$ para a glicemia de jejum, $180 \mathrm{mg} / \mathrm{dl}$ para o valor e 1 hora e de $153 \mathrm{mg} / \mathrm{dL}$ para o valor de 2 horas após sobrecarga com $75 \mathrm{~g}$ de glicose. ${ }^{13}$

Se ao realizar dois destes testes disponíveis em um indivíduo e a resultados forem discordantes, o teste cujo resultado é acima do ponto de corte de diagnóstico deve ser repetido, e o diagnóstico é feito com base na confirmação do teste. Isto é, se um paciente se encontra com a hemoglobina glicada acima dos critérios de diagnóstico e a glicose de jejum normal, ou vive-versa, essa pessoa deve ser considerada diabética através de um novo exame. ${ }^{12}$

O quadro clínico no DM1 é caracterizado pela presença de poliúria, polidpsia, hálito cetônico, polifagia, perda de peso acentuada, glicosúria (decorrente dos níveis glicêmicos que ultrapassam o limiar renal). Esses sintomas podem tanto ocorrer de forma abrupta facilitando o diagnóstico, como também isoladamente, podendo neste caso evoluir para cetoacidose, responsável pelo elevado índice de morbimortalidade. ${ }^{3}$

Já no caso dos pacientes com DM2, 50\% são atendidos no serviço especializado, devido à glicosúria ou à hiperglicemia em exame de rotina. Quadro de poliúria, polidipsia leve e emagrecimento discreto são encontrados em $30 \%$ dos pacientes. Aproximadamente $33 \%$ dos pacientes apresentam cetonúria no diagnóstico e 5\% a 25\% podem evoluir para cetoacidose. A obesidade se apresenta de forma constante no DM2 e sinais clínicos de resistência à insulina também são comuns nesse tipo de diabetes como, acanthosis nigricans, desordens lipídicas (caracterizadas pelo aumento do colesterol total e do LDL colesterol, assim como dos triglicérides) e hipertensão arterial sistêmica (HAS). ${ }^{14}$

A gestação é considerada diabetogênica quando há resistência à insulina, associada ao aumento dos níveis séricos de estrogênio, prolactina, progesterona, cortisol e somatomamotrofina coriônica, visando manter constante o suprimento de glicose para o feto. Na gestação normal isto é compensado pelo aumento da secreção pancreática de insulina. Nas pacientes com alterações no metabolismo dos carboidratos prévias à gestação e nas que não se ajustam às alterações próprias da 
gravidez, a elevação da glicemia materna acarreta hiperglicemia e hiperinsulinêmica fetal, levando a um aumento da morbimortalidade perinatal. ${ }^{15}$

Alguns autores alertam para 0 fato de que mulheres portadoras de intolerância à glicose e hiperglicemia apresentam padrão metabólico alterado com valores mais elevados de IMC, triglicérides, HAS e níveis mais baixos de HDLcolesterol quando comparadas a mulheres normoglicêmicas. ${ }^{8}$

Segundo Isabel ${ }^{\mathrm{n}}$, o DM é uma doença ainda sem tratamento absolutamente satisfatório, estando seu principal problema no desenvolvimento de complicações crônicas, que têm um espectro espantosamente alargado, uma vez que não existe praticamente nenhum órgão ou sistema que não possa ser afetado por esta doença, e dado que as sequelas crônicas do DM podem ter um significativo impacto na vida destes doentes. A presença de complicações crônicas está associada a uma diminuição significativa na qualidade de vida. ${ }^{16}$

O DM é um fator de risco independente para doença arterial coronariana, acidente vascular cerebral, doença vascular periférica e insuficiência cardíaca, que são as principais causas de morte nesses pacientes. Além disso, pacientes com DM e doença cardiovascular têm pior prognóstico, por apresentarem menor sobrevida, maior risco de recorrência da doença e pior resposta aos tratamentos propostos. Os avanços diagnósticos e terapêuticos das últimas décadas já mostram uma redução do risco de eventos cardiovasculares nesses pacientes, mas o risco absoluto desses é ainda duas vezes maior em relação ao dos não diabéticos. ${ }^{17}$

Pacientes com DM1 têm risco duas a quatro vezes maior de desenvolver aterosclerose em relação à população não diabética e, nestes pacientes, os eventos cardiovasculares são responsáveis por até $44 \%$ da mortalidade total. Entretanto, o ganho de peso decorrente do tratamento insulínico intensivo associado às mudanças no padrão alimentar e ao sedentarismo, tem sido relacionado à presença de um perfil lipídico mais aterogênico, com elevação de triglicérides, LDL, ApoB e diminuição de HDL. A prevalência de obesidade, sobrepeso e/ou risco de sobrepeso em pacientes DM1 já atinge $21,2 \%{ }^{18}$

A principal causa de mortalidade no DM1 é a cetoacidose diabética (CAD).Apesar de ser uma complicação inicial relativamente frequente, ela não é exclusiva dessa forma de DM, pois portadores de DM2 também podem abrir seu 
quadro com CAD. Já os estados hiperosmolares hiperglicêmicos (HHS) ocorrem quase exclusivamente no DM2. ${ }^{19}$

Os casos de DM2 são frequentemente oligosintomáticos ou mesmo assintomáticos, podendo um paciente evoluir sem sintomas por vários anos e apresentar apenas ligeira hiperglicemia de jejum ou pós-prandial. No entanto, o DM frequentemente se manifesta por sinais e sintomas das complicações metabólicas agudas ou degenerativas e infecciosas geradas pela doença. A neuropatia diabética raramente é determinante da causa de morte em diabéticos, porém ela é a mais prevalente das complicações crônicas em um serviço geral de assistência a diabéticos (cerca de $40 \%$ dos pacientes têm algum tipo de neuropatia), contribuindo em grande parte para a incapacitação que ocorre nas fases avançadas da doença. ${ }^{20}$

O DM2 é apontado também como uma das principais causas de cegueira entre adultos com idade de 20 a 74 anos. Em alguns estudos, após 15 anos do diagnóstico de DM2, a retinopatia diabética esteve presente em 97\% dos usuários de insulina e em $80 \%$ dos não usuários. A prevalência de nefropatia diabética varia de $10 \%$ a $40 \%$ e a de neuropatia sensitiva distal de $60 \%$ a $70 \%$. Entre os fatores envolvidos na etiologia das complicações crônicas do DM do tipo 2, destacam-se a hiperglicemia, a HAS, a dislipidemia e o tabagismo Além destes, outros fatores de risco não convencionais têm sido descritos: disfunção endotelial, estado prétrombótico e inflamação. ${ }^{21}$

A gestante portadora de DM não tratado tem maior risco de ruptura prematura de membranas, parto prematuro, feto com apresentação pélvica e feto macrossômico. Há também risco elevado de pré-eclâmpsia nessas pacientes. Com relação ao feto, além da macrossomia, o risco para o desenvolvimento de síndrome de angústia respiratória, cardiomiopatia, icterícia, hipoglicemia, hipocalcemia, hipomagnesemia e policitemia com hiperviscosidade sanguínea, encontra-se fortemente aumentado. Em geral, o DM apresenta alta morbimortalidade, com perda importante na qualidade de vida. É uma das principais causas de mortalidade, insuficiência renal, amputação de membros inferiores, cegueira e doença cardiovascular. ${ }^{22}$ 


\section{3 - Terapia Nutricional}

A dieta e o comportamento alimentar estão diretamente associados à prevenção e ao controle do DM. A terapia nutricional (TN) do DM vem sofrendo modificações importantes ao longo do tempo. No início do século 21 a ADA baseouse em evidências científicas nas recomendações nutricionais para DM e desde então tem norteado a conduta nutricional na terapia do DM adotada pela Sociedade Brasileira de Diabetes (SBD).

As diretrizes para o tratamento e acompanhamento do DM da SBD orientam para adoção de plano alimentar saudável, como aspecto fundamental no tratamento do DM, salientando que a orientação nutricional e o estabelecimento de dieta para controle desse grupo de pacientes associados à mudança no estilo de vida são terapias de primeira escolha. ${ }^{23}$

A conduta dietoterápica em pacientes diabéticos deve considerar as fases da vida, diagnóstico nutricional, hábitos alimentares e sócio-econômico-culturais e o requerimento energético baseado nos parâmetros utilizados na população não diabética. ${ }^{12}$

A TN de portadores de DM1 mostra que o adequado controle metabólico tem grande importância por integrar esquemas insulínicos flexíveis ao plano alimentar individualizado e atividade física regular. As recomendações nutricionais atuais, reforçam a importância da implementação de intervenções que minimizem a hiperglicemia pós-prandial.

A qualidade do carboidrato $(\mathrm{CHO})$ da dieta é a maior preditora da glicemia pós-prandial, porém adequadas concentrações desse macronutriente melhoram a sensibilidade à ação da insulina, onde a OMS recomenda a ingestão não inferior a $130 \mathrm{~g} /$ dia. Indivíduos em terapia insulínica tradicional, com doses fixas de insulina, devem ser orientados quanto à importância de respeitar quantidades (gramas ou número de substituições), de $\mathrm{CHO}$ por refeição e horários. ${ }^{24}$

O tipo de $\mathrm{CHO}$ é avaliado de acordo com o índice glicêmico (IG) e a carga glicêmica (CG), sendo o primeiro é a variação sistemática da resposta pós-prandial da glicose e da insulina ao consumo de CHO. Já a CG é a derivação tanto do IG 
como da quantidade de carboidrato ingerida, tendo como objetivo representar o efeito glicêmico da dieta.

Alimentos com baixo IG produzirão menor elevação glicêmica, em comparação com alimentos de alto IG, ${ }^{10}$ além de promover, consequentemente, uma menor demanda de insulina, uma melhora no quadro de lipidemia, e benefícios ligados à prevenção de doenças crônicas como a obesidade, DM e doenças cardíacas. ${ }^{25}$

A quantidade e a qualidade do $\mathrm{CHO}$ consumido, assim como a origem do alimento, forma de preparo, tempo cozimento, teor de gorduras, proteínas e acidez, afetam a resposta glicêmica. Tendo em vista isso, o uso do IG como estratégia nutricional de primeira escolha ainda é controversa, porém tal estratégia pode trazer benefícios adicionais quando o total de $\mathrm{CHO}$ da refeição é contabilizado através de alguns métodos, como a contagem de $\mathrm{CHO}$, o sistema de trocas, cotas ou substituições de alimentos, aliados a experiência individual. ${ }^{24}$

O total de $\mathrm{CHO}$ da dieta é o determinante das doses de insulina do bolus alimentar, no esquema basal-bolus, sendo assim, a razão insulina/CHO pode ser um importante instrumento para nortear os ajustes das doses de insulina às refeições. Observou-se no estudo de DAFNE que pacientes que monitoraram a glicemia préprandial, ajustando a dose de insulina ao $\mathrm{CHO}$, apresentaram sensíveis melhoras nos níveis de hemoglobina glicada, sem aumento significativo nos episódios de hipoglicemia severa. ${ }^{24}$

A conduta nutricional do DM1 não difere do DM2. A sacarose quando consumida em quantidades equivalentes a outros tipos de $\mathrm{CHO}$ não aumenta mais a glicemia em casos de sua ingestão associada a uma dieta saudável, contribuindo, portanto, com $10 \%$ do requerimento energético. Para pacientes que precisam perder peso, a recomendação de sacarose deve ser cuidadosa, já que preparações que a contenha trazem quantidade significativa de gordura. Em relação ao índice glicêmico dos $\mathrm{CHO}$, a ADA considera o método de contagem desse macronutriente a chave da TN no DM1. ${ }^{26}$

Com relação às fibras, a quantidade recomendada deve ser igual a da população geral, indicando-se o mínimo de $20 \mathrm{~g}$ ao dia ou $14 \mathrm{~g}$ por $1000 \mathrm{kcal}$. As fibras solúveis podem interferir na absorção da glicose alimentar diminuindo os níveis glicêmicos pós prandiais. ${ }^{26} \mathrm{O}$ uso de beta-glucanas, fibras altamente 
viscosas, está relacionado à atenuação das respostas glicêmica e insulínica pósprandiais, tendo efeito sobre a degradação do amido e sobre o $\mathrm{CHO}$ disponível e, consequentemente, sobre o IG dos alimentos ingeridos. Sua recomendação tem como objetivo modular a glicemia e a necessidade de insulina, no tratamento da obesidade, doenças cardiovasculares e do DM. ${ }^{24}$

A redução no consumo de gorduras saturadas, trans e de colesterol é o primeiro objetivo para portadores de DM, estando os dois primeiros associados a marcadores inflamatórios e inversamente à sensibilidade à insulina. Segundo as Diretrizes de DM, a recomendação para o colesterol na dieta é inferior a 200mg. Já com relação aos ácidos graxos saturados, por serem os principais determinantes do LDL colesterol, devem contribuir com valores menores que $7 \%$ do valor energético total (VET), enquanto as gorduras trans devem ser minimizadas. Enquanto que os ácidos graxos poliinsaturados devem contribuir com no máximo 10\%. ${ }^{26}$ Baker et al., mostra que a ingestão de 2 gramas de esteróis de plantas e ésteres de etanol, reduzem o colesterol total e o LDL em portadores de DM. ${ }^{27}$

A recomendação de proteína (PTN) para DM é similar ao da população geral, não devendo ultrapassar os valores entre 15 a $20 \%$ do VET (caso a função renal, esteja preservada). Sendo esta oferta proteica constituída de um terço de PTN de alto valor biológico e de proteínas vegetais a fim de suplementar a necessidade de aminoácidos, para síntese e manutenção dos tecidos, além de favorecimento da resposta glicêmica pós-prandial.

O DM é uma doença frequentemente associada à deficiência de micronutrientes, sendo necessário atingir um suporte de vitaminas e minerais diariamente por meio de plano alimentar balanceado. Porém, devido à carência de evidências científicas, a suplementação de antioxidantes, como vitamina $C$ e $E$ não devem ser indicadas. ${ }^{26}$

Os adoçantes são comumente usados na alimentação de indivíduos diabéticos, sendo indicados em substituição a sacarose como objetivo de evitar a ocorrência de picos hiperglicêmicos e reduzir o teor calórico das dietas. ${ }^{10}$ Os edulcorantes liberados para utilização pela população em geral, inclusive para gestantes, são acesulfame K, aspartame, neotame, e sucralose. Recomenda-se o uso moderado de edulcorantes, pois não existem estudos conclusivos, realizados em humanos, sobre sua segurança na gestação. ${ }^{28} \mathrm{Em}$ relação ao aspartame, a 
única contra-indicação é para os portadores de fenilcetonúria, devido a presença de fenilalanina, a qual deve sempre estar especificado nas embalagens dos produtos que a contenha. ${ }^{25}$

Em que concerne o consumo de bebidas alcoólicas, a ADA, em 2009, estabelece a ingestão moderada para mulheres na quantidade de uma dose, e para homem duas doses diárias, devendo-se ter cuidado, pois o excesso no consumo pode induzir a hipoglicemia assim como o consumo de $\mathrm{CHO}$ coingerido com drinques mistos pode elevar a glicemia. ${ }^{25}$

A TN é uma importante aliada no controle do DMG, trazendo benefícios à saúde materno-fetal. Ela deve está focada em escolhas alimentares que garantam um apropriado ganho de peso, controle glicêmico e ausência de corpos cetônicos. ${ }^{29}$ Devido à tendência de formação destes, como consequência do consumo constante de glicose da mãe pelo feto, deve-se evitar períodos de jejum de mais de 4 a 5 horas. $\mathrm{O}$ ganho de peso durante a gestação deve variar de 7 a $16 \mathrm{~kg}$, dependendo do peso pré-gestacional. $O$ aumento nas calorias recomendadas para o $2^{\circ}$ e $3^{\circ}$ trimestres da gestação é de 100 a $150 \mathrm{kcal}$ por dia. Como prescrição inicial, sugerese no início da gestação ou pré-concepção um cálculo de $30 \mathrm{kcal} / \mathrm{kg}$ de peso atual, com aumento para 35 a $38 \mathrm{kcal} / \mathrm{kg}$ dependendo do apetite e ganho de peso da paciente. A ADA sugere $24 \mathrm{kcal} / \mathrm{kg}$ de peso atual para obesas e $12 \mathrm{kcal} / \mathrm{kg}$ para obesas mórbidas. ${ }^{30}$

De acordo com a ADA, as recomendações nutricionais de macronutrientes para gestantes com DM devem ser de $45 \%$ a $65 \%$ do VET para CHO, sendo que fibras solúveis e insolúveis devem ser de 20 a 35g/dia, ou 14g/1000 kcal. Já a recomendação de PTN é de 15 a 20\% do VET, adicionando-se no período gestacional $10 \mathrm{~g}$ ou $1 \mathrm{~g} / \mathrm{kg}$ de peso pré-gestacional/dia, com a ingestão total recomendada de 71g/dia. Em relação aos lipídios, recomenda-se uma ingestão de 20 a $35 \%$ do VET, evidenciando que o consumo de gordura saturada deve ser menor que $7 \%$ do VET e o colesterol menor que $200 \mathrm{mg} / \mathrm{dia}$. Deve-se desestimular também a ingestão de ácidos graxos trans e estimular o consumo de duas a três porções de peixe por semana para o fornecimento de ácidos graxos ômega 3. ${ }^{29}$

O uso de frutooligossacárideos (FOS) vem sendo apontado como uma influência benéfica sobre o metabolismo da glicose. Seus efeitos altamente benéficos podem ser usados na prevenção e controle de doenças particularmente 
associadas com o aumento da resistência periférica à insulina, por exemplo, DM e síndrome metabólica. O uso do suplemento de FOS na dieta é, portanto, uma estratégia para a redução de glicose no sangue, porém a duração do tratamento e da dose a ser administrada são ainda questões que necessitam de estudos. ${ }^{31}$

Mudanças na dieta ajudam a prevenir o desenvolvimento do DM2 e a controlar a glicose sanguínea. Ervas e especiarias também podem ser usadas para controlar a glicose sanguínea, como por exemplo, canela, louro, cravo, noz moscada, avelã, orégano, chá preto e verde, tem sido mostrados como tendo uma atividade biológica "insulin-like". Dessas substâncias, a canela tem demonstrado reduzir a glicose sanguínea, triglicerídeos, colesterol total e frações de LDL em pacientes com DM2, quando adicionada à dieta de 40 dias em doses de 1, 3 ou 6 gramas.

Uma das prováveis explicações da ação da canela no DM seria que o esvaziamento gástrico, dentre outros fatores, regula a resposta da glicose sanguínea pós-prandial e, um retardo na taxa de esvaziamento gástrico, leva a uma diminuição da concentração da glicose sanguínea pós-prandial. Apesar disso, mais pesquisas são necessárias para a canela ser recomendada para o tratamento de pacientes com DM2. ${ }^{32}$

Casas-Agustench $P$ et al.,demonstrou efeitos benéficos do consumo de oleaginosas sobre os lipídios e lipoproteínas plasmáticas. Dentre os componentes das oleaginosas, magnésio, fibras solúveis e insolúveis, ácido graxo alfa-linolênico, L-arginina e antioxidantes podem proteger contra a inflamação e/ou resistência à insulina. A baixa ingestão alimentar e concentração sérica de magnésio foram associados à resistência à insulina e ao DM2.

As fibras solúveis produzem ácidos graxos de cadeia curta, produzidos por fermentação no trato gastrointestinal, contribuindo para diminuir o processo inflamatório. O ácido graxo alfa-linolênico é o precursor da família de ácidos graxos ômega 3, os quais possuem atividade anti-inflamatória e aumentam a sensibilidade à insulina. ${ }^{33}$

Muitos estudos encontraram efeitos benéficos no consumo de oleaginosas sobre a inflamação e a resistência à insulina, outros não observaram nenhum efeito. Tendo em vista a capacidade de componentes nutricionais das oleaginosas em modularem a inflamação, sugere-se que o consumo regular das mesmas associado 
ao estilo de vida saudável possa proteger contra as consequências da inflamação, tais como resistência à insulina e DM2. ${ }^{33}$

\section{4 - CONSIDERAÇÕES FINAIS}

Os dados epidemiológicos mostram a crescente ocorrência de DM bem como o número de óbitos por complicações dessa doença. A importância de se manter o controle da glicemia se faz necessário para evitar tais complicações e a terapia nutricional tem como principal objetivo melhorar a qualidade de vida e prevenir contra as oscilações glicêmicas, evitando desta maneira, as complicações clínicas relacionadas ao DM.

A obtenção de nutrientes adequados a partir de uma dieta equilibrada baseiase nos pilares da pirâmide de alimentação de acordo com a necessidade de cada individuo, proporcionando assim melhora no quadro do portador de diabetes mellitus. O uso de alimentos funcionais e suplementos são indicados para o controle do DM ou para redução das complicações, muitos destes ainda estão em fase de estudos, sendo, portanto necessário cuidado em sua recomendação.

\section{REFERÊNCIAS}

1. Geraldo JM, Alfenas RCG, Alves RDM, Salles VF, Queiroz VMV, Biterncourt MCB. Intervenção Nutricional sobre medidas antropométricas e glicemia de jejum de pacientes diabéticos. Rev. Nutr., Campinas, 21(3); 329-340, maio/jun., 2008

2. Francisco PMSB, Belon AP, Barros MBA, Carandina L, Alves MCGP, Goldbaun M, et al. Cesar CLG. Diabetes auto-referido em idosos: prevalência, fatores associados e práticas de controle. Cad. Saúde Pública, Rio de Janeiro, 26(1):175-184, jan, 2010.

3. Cuppari L.. Nutrição nas Doenças Crônicas Não-transmissivéis. Barueri, SP: Manole, 2009.

4. Mann J.Diabetes Melito e Síndrome Metabólica. In: Mann J, Truswell S. Nutrição Huamana. 2ª Edição. Rio de Janeiro: Guanabara Koogan, 2011

5. Associação Americana de Diabetes. Diabets Basics [Acesso em 2012 Set 20] Disponível em: <http://www.diabetes.org/diabetes-basics/type-2/>

6. Sociedade Brasileira de Diabetes. São 12 milhões de Diabéticos no Brasil [Acesso em 2012 Set 20] Disponível em: < http://www.diabetes.org.br/sala-denoticias/2116-sao-12-milhoes-de-diabeticos-no-brasil> 
7. Pires AC, Chacra AR. A Evolução da Insulinoterapia no Diabetes Melito Tipo 1. Arq Bras Endocrino Metab 2008,52/2

8. Brasil. Diretrizes (2006). Tratamento e Acompanhamento do Diabetes Mellitus. Sociedade Brasileira de Diabetes. Rio de Janeiro-RJ

9. Satorelli DS, Franco LJ. Tendências do diabetes mellitus no Brasil: o papel da transição nutricional. Cad. Saúde Pública, Rio de Janeiro, 19(Sup. 1):S29-S36, 2003

10. Lottenberg AMP. Características da dieta nas diferentes fases da evolução do diabetes melito tipo 1. Arq Bras Endocrinol Metab vol.52 no.2 São Paulo Mar. 2008

11. Brasil. Vigitel (2011). Vigilância de Fatores de Risco e Proteção para Doenças Crônicas por Inquérito Telefônico. Dados sobre Diabetes. SUS Ministério da Saúde

12. American Diabetes Association (2011). Standards of Medical Care in Diabetes. Diabetes care, volume 34, supplement 1, january 2011

13. Farris C. Diagnóstico e rastreamento do diabete melito gestacional. Arq. Catarin. Med. 2012; 41(1): 68-71]

14. Gabby M, Cesarini PR, Dib SA. Diabetes melito do tipo 2 na infância e adolescência: revisão da literatura. Jornal de Pediatria - Vol. 79, №3, 2003

15. Filho FM, Dias CC, Meirelles RS, Cunha SP, Nogueira A, Duarte G. Diabetes e Gravidez: Aspectos Clínicos e Perinatais. Rev. Bras. Ginecol.

Obstet. vol.20 no.4 Rio de Janeiro May 2008

16. Silva I,Pais-Ribeiro J, Cardoso H, Ramos H. Qualidade de vida e complicações crônicas da diabetes. Análise Psicológica (2003), 2 (XXI): 185-194

17. Triches C, Schaan BD, Gross JL, Azevedo MJ. Complicações macrovasculares do diabetes melito: peculiaridades clínicas, de diagnóstico e manejo. Arq Bras Endocrinol Metab. 2009;53/6

18. Matheus ASM, Cobas RA, Gomes MB. Dislipidemias no diabetes melito tipo 1: abordagem atual. Arq Bras Endocrinol Metab vol.52 no.2 São Paulo Mar. 2008

19. Damiani D, Damiani D. Complicações Hiperglicêmicas Agudas no Diabetes Melito Tipo 1 do Jovem. Arq Bras Endrocrinol Metab 2008;52/2

20.Santos ICRV, EF Carvalho, Souza WV, Medeiros MCWC, Nobrega MGL, Lima PMS. Complicações Crônicas dos diabéticos tipo 2 nas Unidade de Saúde da Familia, Recife, Pernambuco, Brasil. Rev. Bras. Saúde Matern. Infant., Recife, 8 (4): 427-433, out, /dez., 2008

21. Scheffel RS, Borlotanza D, Weber CS, Costa La, Canani HL, Santos KG, et al. Crispin D, Roisengerg I, Lisboa HRK, Tres GS, Tschiedel B, Groos JL. 
Prevalência De Complicações Micro E Macrovasculares E De Seus Fatores De Risco Em Pacientes Com Diabetes Melito Do Tipo 2 Em Atendimento Ambulatorial. Rev Assoc Med Bras 2004; 50(3): 263-7

22. Brasília - DF. Cadernos de Atenção Básica (2006). Diabetes Mellitus. Ministério da Saúde

23. Manual de Nutrição Profissional - SBD. Determinando O Plano Alimentar. Cap 3

24. Sociedade Brasileira de Diabetes. Evidências e mitos na terapia nutricional do diabetes mellitus tipo 1 e 2[Acesso em 2012 Out 10] Disponível em: < $<$ http://www.diabetesebook.org.br/modulo-3/24-evidencias-e-mitos-na-terapianutricional-do-diabetes-mellitus-tipo-1-e-2 >

25. Mira GS, Graf H, Cândido LMB. Visão retrospectiva em fibras alimentares com ênfase em beta-glucanas no tratamento do diabetes. Brazilian Journal of Pharmaceutical Sciences vol. 45, n. 1, jan./mar., 2009

26. Brasil. Diretrizes (2009). Diretrizes da Sociedade Brasileira de Diabetes.Tratamento Sociedade Brasileira de Diabetes. [3 ed] Itapevi SP: A Araújo Silva Faramacêutica 2009

27. Baker WL, Baker EL, Coleman C The comparative efficacy of plant sterols and stanols on serum lipids: a systematic review and meta-analysis. 2010 May in Journal of the American Dietetic Association

28. Reis LBSM, Silva APR, Calderon IMP. Acompanhamento nutricional no diabete melito gestacional. Com. Ciências Saúde - 22 Sup 1:S93-S100, 2011

29. Padilha PC, Sena AB, Nogueira JL, Araújo RPS, Alves PD, Accioly E, et al. Saunders C. Terapia nutricional no diabetes gestacional. Rev. Nutr., Campinas, 23(1):95-105, jan./fev., 2010

30. Sociedade Brasileira de Diabetes. Tratamento do diabetes gestacional e da gestante com diabetes [Acesso em 2012 Out 15] Disponível em: <http://www.diabetesebook.org.br/modulo-4/35-tratamento-do-diabetesgestacional-e-da-gestante-com-diabetes>

31. Costa GT, Guimarães SB, Sampaio HAC. Fructo-oligosaccharide effects on blood glucose. Acta Cir. Bras. vol.27 no.3 São Paulo Mar. 2012

32. Sociedade Brasileira de Diabetes Efeito da canela na glicemia pós-prandial, esvaziamento gástrico e saciedade em pessoas saudáveis.[Acesso em 2012 Out 15] Disponível em: <http://www.diabetes.org.br/colunistas-da-sbd/nutricao-eciencia/1017> 
33. Casas-Agustench $P$, Bulló $M$, Salas-Salvadó J. Nuts, inflammation and insulin resistance. Asia Pac J Clin Nutr 2010;19 (1):124-130 